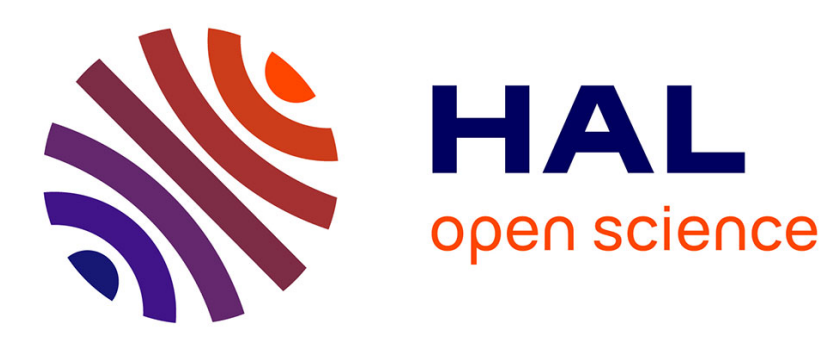

\title{
On the content of sensorimotor representations after actual and motor imagery practice
}

Lucette Toussaint, Nicolas Robin, Yannick Blandin

\section{To cite this version:}

Lucette Toussaint, Nicolas Robin, Yannick Blandin. On the content of sensorimotor representations after actual and motor imagery practice. Motor Control, 2010, 14 (2), pp.159-175. 10.1123/mcj.14.2.159 . hal-01540065

\section{HAL Id: hal-01540065 \\ https://hal.science/hal-01540065}

Submitted on 9 Apr 2020

HAL is a multi-disciplinary open access archive for the deposit and dissemination of scientific research documents, whether they are published or not. The documents may come from teaching and research institutions in France or abroad, or from public or private research centers.
L'archive ouverte pluridisciplinaire HAL, est destinée au dépôt et à la diffusion de documents scientifiques de niveau recherche, publiés ou non, émanant des établissements d'enseignement et de recherche français ou étrangers, des laboratoires publics ou privés. 


\title{
On the Content of Sensorimotor Representations After Actual and Motor Imagery Practice
}

\author{
Lucette Toussaint, Nicolas Robin, \\ and Yannick Blandin
}

\begin{abstract}
We examined the similarities between actual and motor imagery practice with regard to the development of sensorimotor representations. Participants had to reproduce knee joint positions (15 or 150 trials) in visuo-proprioceptive or proprioceptive conditions (Experiment 1) or in visual, proprioceptive or visuoproprioceptive imagery conditions (Experiment 2), before being transferred in a proprioceptive condition. A familiarization session in a proprioceptive condition was performed before imagery practice only (Experiment 2 ). Results showed that the effect of vision withdrawal varied according to actual or motor imagery practice: performance accuracy in transfer decreased after actual visuo-proprioceptive practice while it increased after visuo-proprioceptive imagery practice. These results suggest that different movement representations can be developed following actual or imagery practice. They also suggest that information from previous experience could be stored in a sensori-motor memory and could be fundamental for the efficiency of motor imagery practice.
\end{abstract}

Keywords: exercise, motor learning, motor performance, specificity of practice.

Behavioral and brain mapping studies reveal a close relationship between actual and imagined actions. For example, positron emission tomography and functional magnetic resonance imaging researches showed that similar cortical regions are involved in both actual and mental simulation of a movement (Decety, Perani, Jeannerod, Bettinardi, Tadary, Woods et al., 1994; Miyai, Tanabe, Sase, Eda, Oda, Konishi et al., 2001; Stippich, Ochmann \& Sartor, 2002). Furthermore, similarities between actual and simulated actions have also been shown with behavioral studies, where the time course of a mentally simulated movement appeared positively correlated to its actual duration (Decety \& Michel, 1989; Georgopoulos \& Massey, 1987; Jeannerod, 1994) as well as with measurements of autonomic responses (Guillot \& Collet, 2005; Decety, Jeannerod, Germain \& Pastene, 1991) and the changes of excitability of spinal reflex pathways (Roure, Collet, DeschaumesMolinaro, Delhomme, Dittmar \& Vernet-Maury, 1999). In the current study, we questioned the similarity between actual and imagined actions by comparing motor performance following these two forms of practice. More specifically, we examined

The authors are with CeRCA, CNRS UMR 6234, Poitiers, France. 
whether similar sensorimotor representations were developed through actual and motor imagery practice.

Over the last thirty years, some works examining the specificity of learning hypothesis (Proteau, 1995; Proteau, Marteniuk, Girouard \& Dugas, 1987; Proteau, Marteniuk \& Lévesque, 1992) argued that motor learning is specific to the source(s) of information that are more likely to ensure optimal performance (Proteau \& Carnahan, 2001; Robin, Toussaint, Blandin \& Vinter, 2004; Robin, Toussaint, Blandin \& Proteau, 2005; Soucy \& Proteau, 2001; Tremblay \& Proteau, 1998). The majority of studies using various tasks (aiming, locomotion, powerlifting) have shown the domination of visual information over proprioception during motor practice (Ivens \& Marteniuk, 1997; Mackrous \& Proteau, 2007; Proteau \& Carnahan, 2001; Proteau, Tremblay \& DeJaeger, 1998; Soucy \& Proteau, 2001). This is especially true when visual information was sufficiently salient and allowed better movement accuracy than other source of afferent information (Robin et al., 2005; Tremblay \& Proteau, 1998). For example, Proteau and collaborators (1987, 1992, 2001) observed that during video-aiming, participants who practiced the task with both visual and proprioceptive information (i.e., VP condition) were less accurate in a transfer test, when they had to perform the same task in a proprioceptive only condition (P condition), than participants who practiced it in a proprioceptive (P) condition. Moreover, the withdrawing of visual information on movement execution in transfer resulted either in a large deterioration in motor performance early during learning (i.e., after 20 trials; Tremblay \& Proteau, 1998) or in a deterioration that increased with practice (Blandin, Toussaint \& Shea, 2008; Ivens \& Marteniuk, 1997; Krigolson, Van Gyn, Tremblay \& Heath, 2006; Proteau et al., 1987, 1992, 1998). Vision can thus be considered as the dominant sensory information for motor learning and control

This dominance of visual cues was challenged in other works (Tremblay, Welsh \& Elliott, 2001; Robin et al., 2004, 2005). Tremblay and collaborators (2001) studied aiming toward continually visible targets located straight ahead and aligned with the sagital axis of the participant's body. Four groups performed either 15 trials or 150 trials under either a constant or a variable practice schedule with both visual and proprioceptive information (VP condition) or with only proprioceptive information ( $\mathrm{P}$ condition) during acquisition. Results showed that aiming accuracy in transfer ( $\mathrm{P}$ and VP conditions) increased with practice, especially for groups which completed the acquisition phase under a variable practice schedule. Contrary to studies mentioned in the precedent section, these results rather showed that the utilization of on-line proprioceptive information was enhanced through practice regardless of the availability of vision during acquisition. The dominance of the visual cues was also challenged when only ambient visual information was available during powerlift squat (i.e., no visual dynamic information on movements, Tremblay \& Proteau, 1998), for pointing tasks toward "self-defined" targets (Robin et al., 2004) or in a weak vision condition (Robin et al., 2005). In the latter studies, for example, it appears that less salient visual cues available in the weak vision (the color of the cursor contrasted little with the background of the screen) compared with the normal vision condition did not mask the processing of other sources of afferent information, but contrary to strong visual cues helped participants calibrate less salient sources of afferent information such as proprioception. It thus appeared that if the superiority of one source of afferent information cannot be established 
easily, the CNS processes other source of afferent information available to ensure performance accuracy. Overall, these studies showed that the dominance of visual information for motor control and learning can be modulated by the task constraints.

To our mind, the importance of sensory modalities available during motor learning is still an interesting question with regard to other types of practice such as motor imagery practice. Mentally performing a motor task generally has a positive effect on learning (Driskell, Cooper \& Moran, 1994; Féry, 2003; Gentili, Papaxanthis \& Pozzo, 2006; Papadelis, Kourtidou-Papadeli, Bamidis \& Albani, 2007; Yue \& Cole, 1992). However, several questions remain to determine the optimal conditions to be used to improve learning. The present set of experiments explored whether the effect of the sensory modalities (visual, proprioceptive) available during motor learning are similar in actual practice and motor imagery practice. For that purpose, participants were placed in sensory-specific learning conditions during actual practice (i.e., a visuo-proprioceptive or a proprioceptive condition, Experiment 1) and received sensory-specific motor imagery instructions for mental practice in Experiment 2 (visual, proprioceptive or visuo-proprioceptive imagery), after having first participated to a familiarization session of the task.

In a recent work, Krigolson and collaborators (2006) questioned the similarities between actual and motor imagery practices on the development of a sensoryspecific movement representation during a walking task. Participants were divided into either visuo-proprioceptive (VP), proprioceptive only $(\mathrm{P})$ or visual imagery (VI) practice groups during an acquisition phase and were then transferred to an actual practice test performed in a $\mathrm{P}$ condition. Analysis of walking accuracy showed that participants for both the actual and imagined conditions were more accurate when the sensory information remained similar from acquisition to transfer when compared with a condition where sensory information was withdrawn. For the authors, result suggested that one can become dependent upon an imagined source of visual information in the same manner as one can become dependent upon actual sensory information. Such results were of primary importance because they showed that the development of a sensory-specific movement representation may take place after either actual or mental practice. In the current study, in line with Krigolson et al.'s (2006) work, we used the specificity of practice hypothesis to examine the content of sensorimotor representations developed through imagery practice. In particular, we asked whether motor imagery practice could also induce the superiority of a specific source of information over other sources of information available during practice to ensure motor performance accuracy, as reported for actual practice. Note that in Krigolson and collaborators' experiment (2006) only a visual imagery group was compared with actual practice.

In the first experiment, we examined whether learning of simple body configuration reproduction is specific to visual information. Participants had to reproduce knee joint positions in either a visuo-proprioceptive condition (VP) or in a proprioceptive condition only $(\mathrm{P})$. Following 15 and 150 trials of practice, all participants were transferred to a proprioceptive $(\mathrm{P})$ condition without Knowledge of Results. In agreement with the specificity of practice hypothesis, visual dominance should induce less accurate body configuration reproduction in transfer for the visuoproprioceptive (VP) than for the proprioceptive (P) group. Furthermore, if visual dominance increases with practice, the deleterious effect of vision withdrawal in transfer (VP group) should be more important after 150 trials of practice than after 
15 trials. By contrast, if the role played by visual information on body configuration reproduction decreases with practice, the withdrawing of vision in transfer should be more detrimental to performance accuracy after 15 trials of practice than after 150 trials. In Experiment 2, the specificity of practice paradigm was applied with 15 and 150 motor imagery trials (i.e., visual, proprioceptive or visuo-proprioceptive imageries). The experimental procedure differed slightly from Experiment 1, participants having first to perform a familiarization session to experience each of the body configurations to be mentally reproduced during the acquisition phase.

\section{Experiment 1}

\section{Method}

Participants. Twenty four students (mean age $=23.6, S D=2.8$ ) participated on a volunteer basis in the experiment. All were self-declared right-footed, healthy, without history of past or present nervous or muscular disorders. All were naïve to the experimental task and conditions and gave their informed consent prior to the beginning of the experiment. This experiment has been approved by the Local Ethics Committee.

Task and Apparatus. Participants were seated comfortably on a table so that their left leg could move freely around the knee joint. A goniometer, equipped with a potentiometer $\left(\right.$ Sfernice, $5 \mathrm{~K} \Omega 0127$, resolution $=0.07^{\circ}$ ), was applied on their left leg so that the axis of rotation of the goniometer corresponded to the rotation axis of the left knee joint. The values recorded when participants were seated on the table, their leg in the edge, was used as the reference value (around $90^{\circ}$ ) and corresponded to the resting position for a specific experimental phase.

A typical trial of the experimental phases was divided in two phases. First, during the encoding phase, participants were asked to actively stretch out their left leg to reach one of the 3 angular positions chosen in this study $\left(132^{\circ}, 147^{\circ}\right.$ or $\left.162^{\circ}\right)$. Their leg was stopped by the experimenter when it reached the required position. This position was maintained $2 \mathrm{~s}$ and participants repositioned their leg to the resting position. In the second phase, recall phase, participants had to reproduce as accurately as possible the position previously reached, without speed requirements.

Procedure. Participants performed successive acquisition and transfer phases. During acquisition, participants performed the task in a visuo-proprioceptive (VP) or a proprioceptive $(\mathrm{P})$ condition. In the visuo-proprioceptive condition, they could see their left leg in a mirror located on their left during both encoding and recall positions. The mirror was placed on the side of participants to provide accurate feedback on angular position of the leg. An opaque black cloth was placed around the table where participants were seated (ahead, behind and on their right) to withdraw the vision of other environmental cues. In the proprioceptive condition, vision of the leg was occluded, participants being instructed to close their eyes. However, vision of the leg in the resting position was allowed before each trial to update the proprioceptive map. In the first acquisition phase, participants had to perform 15 trials ( 5 random trials for each 3 positions in the VP or $\mathrm{P}$ condition), while in the second acquisition phase, they performed 150 trials (50 random trials for each 3 positions) in a similar sensory condition as the previous one. 
Knowledge of results was provided following each trial. It indicated differences (degrees) between encoding and recall positions.

Ten minutes after each acquisition phase, participants were transferred to a $\mathrm{P}$ condition. They had to perform 15 trials ( 5 random trials for each 3 positions) with their eyes closed and without knowledge of results. All participants could see their leg in the resting position before each trial.

\section{Data Analysis}

Angular difference between encoding and recall positions was computed for each trial. Because no systematic difference between positions (overshoot or undershoot) was reported, data were grouped into blocks of 15 trials for each experimental condition. Constant (CE) and variable error (VE) were then computed. However, because statistical analyses revealed similar results between these two measures, they were combined and analyzed as root mean square errors (RMSE). Details of the statistical analyses will be provided at the beginning of each subsection of the results. In all cases, significant main effects and interactions were broken down by using the Newman-Keuls technique. Alpha was set at .05 for all analyses (with Bonferroni adjustments).

\section{Results}

\section{Acquisition}

In this section, we contrasted the participant's performance in acquisition as a function of the sensory conditions. RMSE was submitted to an ANOVA contrasting 2 Condition $(\mathrm{VP}$ and $\mathrm{P}) \times 11$ Block (block 1 for the 15 acquisition trials, blocks 2-11 for the 150 acquisition trials) with repeated measures on the last factor.

The statistical analysis showed an effect of Block, $F(10,220)=16.41$, MSE $=0.21, p<.001$, but there was no effect of Condition, $F(1,22)=0.90$, MSE = 4.10 , and no interaction, $F(10,220)=0.55, \mathrm{MSE}=0.205$. As illustrated in Figure 1 (Top panel), RMSE decreased as a function of practice, whatever the sensory conditions. Post hoc comparisons indicated significant differences between the first block (15 acquisition trials) and the other blocks, as well as difference between the first 3 blocks of the 150 acquisition trials phase (blocks 2-4) and the last two blocks (blocks 10 and 11).

\section{Acquisition versus Transfer}

Blocks 1 and 11 of the acquisition phases were retained as representative of participants' performance at the end of each acquisition phase (15 trials and 150 trials of acquisition respectively) and performance was contrasted to the one obtained in transfer. RMSE was submitted to a 2 Condition (VP and P) $\times 2$ Acquisition (15 trials and 150 trials) $\times 2$ Experimental phase (end of acquisition and transfer) with repeated measures on the last two factors.

The ANOVA revealed an effect of Acquisition, $F(1,22)=41,84, M S E=0.37$, $p<.001$, and of Experimental phase, $F(1,22)=13.87, M S E=0.22, p<.001$, as well as significant interactions between Condition $x$ Experimental phase, $F(1,22)$ 


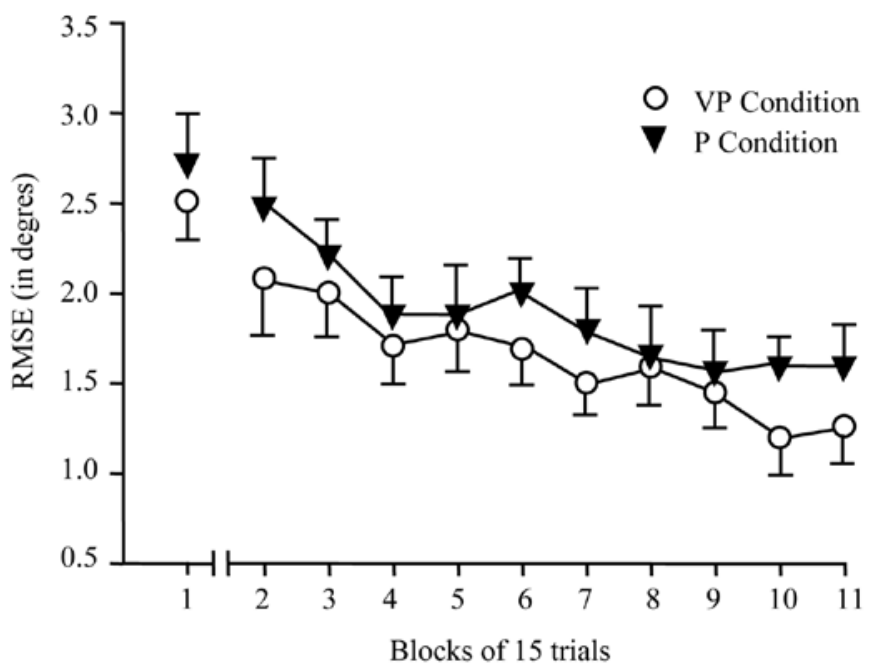

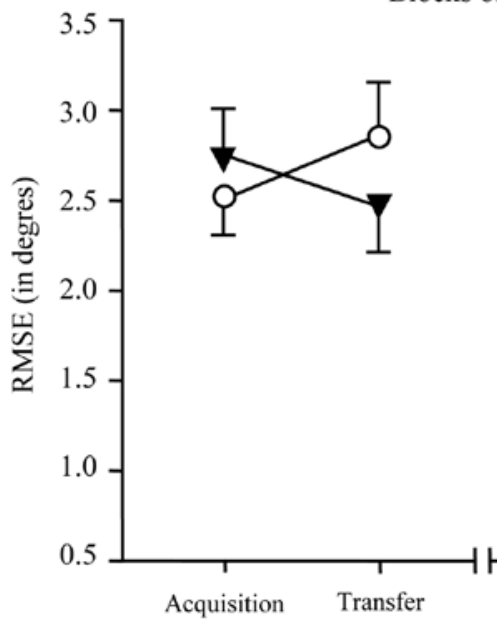

15 practice trials

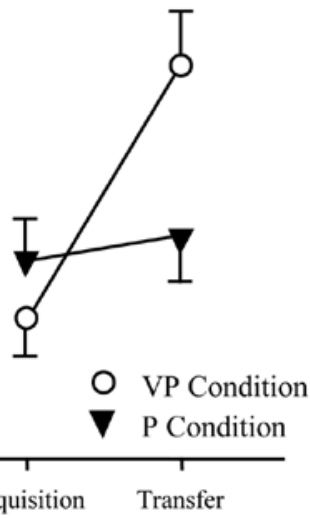

150 practice trials

Experimental phases

Figure 1 - Top panel: RMSE as a function of sensory conditions (VP and P) and blocks of practice. Block 1 corresponded to the first acquisition phase (15 trials), blocks $2-11$ corresponded to the second acquisition phase (150 trials). Lower panel: RMSE as a function of sensory conditions (VP and P), acquisition (15 trials and 150 trials) and experimental phases (acquisition and transfer).

$=24.83, M S E=0.22, p<.001$, Acquisition $x$ Experimental phase, $F(1,22)=11.85$, $M S E=0.24, p<.01$, and Condition $\mathrm{x}$ Acquisition $\mathrm{x}$ Experimental phase, $F(1,22)$ $=7.46, M S E=0.87, p<.01$. There was no effect of Condition, $F(1,22)=0.78$, $M S E=1.44$, and no Condition $\mathrm{x}$ Experimental phase interaction, $F(1,22)=0.33$, $M S E=0.37$. The breakdown of the Condition $\mathrm{x}$ Acquisition $\mathrm{x}$ Experimental phase 
interaction revealed that RMSE remained stable from acquisition to transfer after 15 acquisition trials whatever the acquisition condition. By contrast, RMSE significantly increased in transfer after 150 acquisition trials under visuo-proprioceptive condition (VP), while it did not vary under proprioceptive condition (P). Note that after 15 practice trials RMSE was lesser at the end of acquisition for participants who practiced under visuo-proprioceptive condition than under proprioceptive condition, while the reverse was true in transfer (see Figure 1, Bottom panel).

\section{Discussion}

The purpose of the first experiment was to examine the applicability of the specificity of practice hypothesis to a simple body configuration reproduction task. In manual aiming toward visual targets, it has been proposed that early in practice, participants are able to determine the source(s) of afferent information that are more likely to ensure optimal accuracy, this role being mainly devoted to visual feedback processing (Coull, Tremblay \& Elliott, 2001; Ivens \& Marteniuk, 1997; Mackrous \& Proteau, 2007; Proteau \& Carnahan, 2001; Tremblay \& Proteau, 1998). The increased reliance upon visual feedback has also been supported by study of gross motor tasks as walking (Krigolson et al., 2006; Proteau et al., 1998), powerlifting (Tremblay \& Proteau, 1998) and sequential positioning (Ivens \& Marteniuk, 1997). However, others studies challenged the visual dominance in motor control and learning, the detrimental effect of vision withdrawal decreasing with practice (Robin et al., 2004, 2005; Tremblay et al., 2001). In the present experiment, we asked whether visual dominance appears or not when participants have to reproduce simple body configurations.

The main result of the present experiment showed that vision of the movement leg in the mirror plays a dominant role in body configuration reproduction after a sufficient amount of practice. At the end of the 150 trials acquisition phase, motor performance was more accurate for the visuo-proprioceptive group than for the proprioceptive group. Moreover, the withdrawal of visual feedback in transfer leaded to increased RMSE when compared with RMSE recorded at the end of acquisition phase. Note that this reliance upon visual cues did not appear after 15 acquisition trials where motor performance accuracy was similar in acquisition and transfer for both sensory conditions of practice (VP or P conditions). These results suggest that, contrary to a lot of studies where visual dominance was reported early in practice, determining that visual information is more likely to ensure optimal accuracy is time consuming with regard to angular body production.

The early or late reliance upon vision could depend on the task constraints. Aiming toward a continuously visible target corresponded to movement performed in an allocentric frame of reference, where accuracy of movement required simultaneous vision of the arm and the target (Proteau \& Carnahan, 2001). By contrast, in everyday life, vision of a relevant body part is not always available and reproduction of simple body configurations required the processing of egocentric information, based on body positions. Shenton, Schwoebel \& Coslett (2004) reported that under some circumstances (i.e., without requirement of spatial aim) proprioception represented the dominant sensory input to the on-line representation of the body in space. It was not the case after 150 trials of practice in our present experiment. To our mind, it may be possible that the unusual utilization of a mirror to provide 
visual information on the body positions constrains participants to progressively learn to use visual information to increase the accuracy of body configuration reproductions. This unusual information would give some complementary cues on angular accuracy of the knee by comparison with sensory cues provided by proprioception alone, and would necessitate that participants acquire a sufficient level of expertise to be correctly used.

The main results of the present experiment agree with one prediction of the specificity of learning hypothesis: practice leads to the development of a sensoryspecific movement representation (Proteau et al., 1987; Krigolson et al., 2006). We effectively observed that participants who practiced in a sensory condition that matched the sensory conditions of transfer (with proprioception only) were more accurate than participants who practiced under a dissimilar sensory condition (with both vision and proprioception). When many sensory modalities were available, as in the visuo-proprioceptive condition, reliance upon a particular source of feedback (vision in the current study) is time-consuming and appeared only later during practice.

\section{Experiment 2}

In the second experiment, we examined whether motor imagery practice leads to the development of a sensory-specific movement representation similar to that developed under actual practice. After a familiarization session of the task with physical practice, participants were instructed to mentally reproduce knee joint positions in either visual imagery (VI), proprioceptive imagery (PI) or visuoproprioceptive imagery (VPI) conditions. In the latter condition, participants performed simultaneously visual and proprioceptive imageries. Following 15 and 150 imagery trials, participants performed transfer trials in a proprioceptive condition (P condition) without Knowledge of Results. In accordance with the specificity of practice hypothesis and with the idea of functional equivalence between actual and motor imagery practice, we predicted that the proprioceptive imagery group (PI) should be more accurate in transfer than the visual imagery (VI) and visuoproprioceptive imagery (VPI) groups. Furthermore, the accuracy in transfer should increase as a function of practice for the proprioceptive imagery group, whereas it should decrease in the visual imagery and visuo-proprioceptive imagery groups due to the increased reliance upon visual information. More important in the present work are the results obtained for the visuo-proprioceptive imagery group. The applicability of specificity of learning hypothesis to motor imagery practice, that is the prominent role of vision in movement planning and execution, should be manifest in transfer by a decrease of movement accuracy for both the visual imagery and visuo-proprioceptive imagery groups.

\section{Method}

Participants. Thirty students (mean age $=22.7, S D=2.4$ ) participated on a volunteer basis in the experiment. None of them took part into Experiment 1. All were self-declared right-footed, healthy, without history of past or present nervous or muscular disorders. They were also retained on the basis of their scores on the Movement Imagery Questionnaire (MIQ, Hall \& Pongrac, 1983). This 
questionnaire measures the difficulty of forming visual and proprioceptive images of simple physical movements with a Likert-type scale. In the current study, only participants with a score inferior to 19 for both visual and kinesthetic imageries were retained because they can be considered as high imagers. All of them were naïve to the experimental task and conditions and gave their informed consent prior the beginning of the experiment. This study has been approved by the Local Ethics Committee.

Task and Procedure. The apparatus was similar to the one described in the first experiment. A typical trial was divided into successive encoding and recall positions. In the present experiment, because motor imagery practice required a minimal exposure to the task, participants performed 5 familiarization trials for each position $\left(132^{\circ}, 147^{\circ}\right.$ or $162^{\circ}$ random presentation) with KR but without vision (i.e., in the same sensory condition used in transfer tests). Then, participants performed two successive acquisition and transfer phases.

During the first acquisition phase, participants performed 15 trials of motor imagery practice ( 5 random trials per positions) in either visual imagery (VI), proprioceptive imagery (PI) or mixed imagery (VPI) conditions. In the visual imagery condition, participants had to imagine themselves reaching the position asked by the experimenter as accurately as possible, focusing on visual information as provided by the mirror (located at their left, as for Experiment 1). In the proprioceptive imagery (or kinesthetic imagery) condition, they were asked to feel themselves reaching the position as accurately as possible, focusing on the bodily information. In the visuo-proprioceptive imagery condition, participants were asked to simultaneously visually imagine and feel themselves reaching the position (see Appendix A for details on imagery scripts).

For each participant, the second acquisition phase consisted to mentally simulate 150 trials ( 50 random trials for each 3 positions) in a sensory condition similar to the one used during the first acquisition phase (VI, PI or VPI conditions). During motor imagery, for the first and second acquisition phases, participants were seated on the table with the legs in the resting position.

Ten minutes after each acquisition, participants were transferred in a P condition. They had to perform 15 trials ( 5 random trials for each 3 positions) with their eyes closed and without knowledge of results. All participants could see their leg in the resting position before each trial.

\section{Data Analysis}

Angular difference between encoding and recall positions was computed for each trial recorded during transfer. As in Experiment 1, because no systematic differences between position (overshoot or undershoot) were reported, data were grouped into blocks of 15 trials. Because statistical analyses revealed similar results between constant (CE) and variable error (VE), they were combined and analyzed as root mean square error (RMSE). RMSE was submitted to an ANOVA contrasting 3 Condition (VI, PI, VPI) $\times 2$ Acquisition (15 trials and 150 trials) with repeated measures on the last factor. Significant main effects and interactions were broken down by using the Newman-Keuls technique (alpha =.05). Bonferroni adjustments of the p-values were used. 


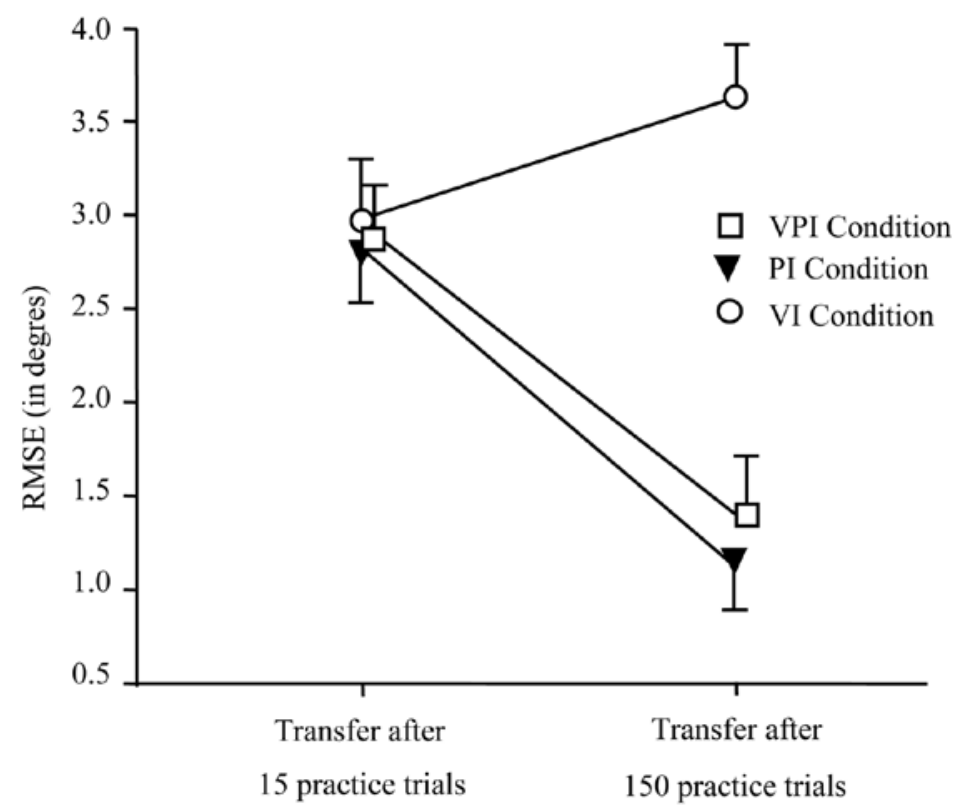

Figure 2 - RMSE as a function of imagery conditions (VI, PI and VPI) and acquisition (15 trials and 150 trials) recorded during the transfer phases.

\section{Results}

The ANOVA revealed significant main effects of Condition, $F(2,27)=13.62, M S E$ $=0.93, p<.0001$ and of Acquisition, $F(1,27)=35.15, M S E=0.35, p<.0001$, as well as a significant interaction between these two factors, $F(2,27)=23.90, M S E$ $=0.35, p<.0001$. As illustrated in Figure 2 and confirmed by post hoc analyses, RMSE significantly increased with practice in the visual imagery condition (VI), while it decreased in the proprioceptive imagery (PI) and visuo-proprioceptive imagery (VPI) conditions. This led to significant differences between the visual imagery condition and the two others (PI and VPI condition) after 150 imagery trials, while performances were similar in the three conditions after 15 imagery trials.

\section{Discussion}

The aim of the second experiment was to use the specificity of practice paradigm to examine whether imagery practice led to the development of a sensory-specific movement representation. In agreement with the specificity hypothesis, we predicted that participants' performance in transfer would be determined by the similarity of the sensory modality between the acquisition and transfer conditions. A significant reduction in motor performance should be observed when sensory information used during mental practice (VI and VPI conditions) does not match sensory information 
available during transfer ( $\mathrm{P}$ condition). By contrast, sensory modality similarity between imagery practice (PI condition) and transfer (P condition) should lead to optimal motor performance. Moreover, the expected process of visual information to the detriment of other sources of information should be manifested by a similar performance between VI and VPI conditions.

The overall results were only partially in agreement with our predictions. Similarities of sensory information between acquisition (PI condition) and transfer (P condition) led effectively to optimal accuracy of knee positions. By contrast, when acquisition was performed with a sensory modality (VI condition) that did not match the one available in transfer (P condition), performance suffered from this discrepancy. Note that performance discrepancy between transfer tests performed after 15 and 150 trials of practice was not observed in the Experiment 1 (i.e., The VP group did not exhibit larger RMSE after 150 versus 15 acquisition trials, while the VI group did. See also Footnote 1). The random presentation of target knee angles employed in our study could explain that RMSE in transfer did not increase with practice for the VP group, a variable practice schedule promoting the utilization of on-line proprioceptive information when both visual and proprioceptive cues are available (Tremblay et al., 2001). Similar results have been previously reported by Robin, Toussaint \& Blandin (2006) after 90 trials of visual or proprioceptive motor imagery practice in a similar task and authors highlighted the necessity to maintain constant the dominant sensory modality of the task and the imagery modality used in mental practice (i.e., proprioception). However, their conclusion was only based on data recording after motor imagery practice. In the current study, we also examined motor performance improvements after actual practice in various sensory conditions (Experiment 1). The reliance on visual information reported after 150 trials of motor practice for that specific task appears inconsistent with the idea that proprioception is the dominant sensory modality in a body configuration reproduction task. Consequently, the dominance of proprioceptive motor imagery on knee joint position reproduction improvements cannot be explained by sensory dominance processes. We will come back to this point in the general discussion section.

Contrary to our hypothesis based on the specificity of learning hypothesis, performance in transfer for participants in the visual and proprioceptive condition (VPI) was similar to performance recorded in the proprioceptive imagery condition (PI). This result disagreed with the initial tenet of the specificity of practice hypothesis (Proteau et al., 1987) predicting that practice led to the development of an intermodal representation, a movement representation that strongly integrated all sensory information available during acquisition. In that case, the performance should suffer if one sensory source of information integrated in the movement representation is lacking. Clearly, it is not the case in the present experiment. The withdrawal of vision in transfer after visuo-proprioceptive imagery practice did not induce performance decrement.

This similarity between proprioceptive imagery and visuo-proprioceptive imagery conditions would rather be in agreement with a more recent conception of the specificity of practice hypothesis: with practice, participants are able to determine the source of sensory information that are more likely to ensure optimal accuracy and to process it to the detriment of all others (Coull et al., 2001; Proteau \& Carnahan, 2001; Tremblay \& Proteau, 1998). With regard to simple body configuration 
reproduction, performance recorded for participants who had to simultaneously visually imagine and feel themselves reaching a specific position (VPI condition) could indicate that they favored the processing of proprioceptive information to the detriment of visual information. When reproducing simple body configurations, practice in the VPI condition would thus result in the development of a movement representation reliant upon imagined proprioceptive feedback, afferent information that ensures optimal accuracy, when compared with results obtained for imagined visual feedback (VI condition).

\section{General Discussion}

In conclusion, the present results are partially in line with the specificity of learning hypothesis developed for aiming and confirmed for various motor tasks. Both actual and mental practice leads to the development of a sensory-specific movement representation reliant upon a specific source of sensory information. These results are consistent with the work of Krigolson and collaborators (2006) which showed that when participants became dependent upon a source of information (actual or imagined visual information), its withdrawal in a transfer condition demonstrated greater movement error than those who did not. However, in the current study, we demonstrated that the movement representation developed when both visual and proprioceptive information are available during acquisition could differ following actual or motor imagery practice. The first experiment on actual practice showed the dominance of visual information after 150 trials of practice. By contrast, the second experiment on mental practice rather showed an increased reliance upon proprioceptive information.

The discrepancy between actual and motor imagery practice with regard to sensory dominance may be explained in two ways. First, only proprioceptive motor imagery practice would induce changes in motor performance similar to those observed following actual practice ${ }^{1}$. Using a transcranial magnetic stimulation, Stinear, Byblow, Steyvers, Levin and Swinnen (2006) compared the modulation of primary motor cortex activation during proprioceptive motor imagery and visual motor imagery of phasic thumb movements. Only proprioceptive imagery modulated corticospinal exitability in a way similar to actual practice. The authors concluded that emphasis should be placed on proprioceptive rather than visual motor imagery in sensory-motor learning and rehabilitation. However, Stinear and collaborators' point of view should be interpreted with caution because behavioral studies have effectively reported the beneficial effect of visual motor imagery practice in skill learning (Féry, 2003; Krigolson et al., 2006; Noel 1980; Robin, Dominique, Toussaint, Blandin, Guillot \& Le Her, 2007).

Second, the sensory conditions of the task experimented before mental practice could explained the difference between actual and motor imagery practice. Because there was a proprioceptive experience prior the motor imagery acquisition phase (i.e., during the familiarization phase in Experiment 2), the recent proprioceptive feeling for the body configuration reproduction may have been more easily incorporated in the participants' movement representation. This previous proprioceptive exposure probably led to the process of proprioception to the detriment of the other sources of information available during acquisition (i.e., vision in the present experiment). We thus suggest that the development of a sensorimotor representa- 
tion during mental practice could be specific to the recent sensory experience of the task (i.e., sensory conditions available immediately prior imagery practice).

Moreover, our data could suggest that to successfully employ motor imagery, it is critical that participants have previously developed a sensory-specific representation of actions. This suggestion is supported by recent fRMI studies that showed that although physical activity and motor imagery recruit common brain activation, the neural networks underlying different imagery modalities (visual, kinesthetic/ proprioceptive) are not identical (Binkofski, Amunts, Stephan, Posse, Schormann, Freund et al., 2000; Guillot, Collet, Nguyen, Malouin, Richards \& Doyen, 2009; Solodkin, Hlustik, Chen \& Small, 2004). In a finger movement task, Guillot et al. (2009) directly compared brain activation following visual and kinesthetic imagery in the same group of good imagers. They reported neuroanatomical evidence of neural networks with regard to the use of motor imagery based on specific sensory cues. It thus appeared that visual and kinesthetic motor imagery of finger movements are mediated by neural substrats involving the cerebral regions related to the predominant sensory systems supporting the motor imagery content. Their findings corroborate behavioral studies on the selectively effects of imagery modalities during motor imagery practice with regard to the characteristics of the motor skill (Guillot \& Collet 2008, for a review). Our results indicated that the sensory-specific representation developed before imagery practice has also to be taken into account to explain selectively effects of imagery modalities during motor imagery practice. In that case, the specificity of practice effect following motor imagery could be conceived as the rehearsal of a preexisting sensory-motor representation, while physical practice both generates and enhances a sensory-specific motor representation as a function of the more accurate sensory cues that are more likely to ensure optimal performance.

How motor learning occurs following motor imagery is still a matter of some debate. According to the central theory, motor learning following motor imagery practice could be attributed to the solicitation of similar cognitive mechanisms as in physical practice (Jeannerod, 2001). The notion that a sensory motor memory of a previously executed movement is stored in the CNS and retrieved to internally simulate the same movement, as suggested in the previous section, is in agreement with the theory of internal models (Wolpert \& Kawato, 1998). When participants brought their leg in a specific angular position, the inverse internal model generated the appropriate motor command corresponding to the desired position. The forward model predicted the future states of the leg and the sensory consequence of that particular movement. During motor imagery, the execution of the motor command is blocked, and no sensory information arises from periphery. However, the information stored in the sensory motor memory after the execution of the actual movement is retrieved and used to simulate the same movement (forward process). This motor prediction would strictly depend on the sensory motor memory of the task executed before mental rehearsal: a proprioceptive motor memory developed during the familiarization phase in the second experiment of the current study. Motor improvement following motor imagery practice would be due to sensory motor predictions making by the forward model (Flanagan, Vetter, Johansson \& Wolpert, 2003), the accuracy of these predictions being highly dependent on the participants' previous sensory experience. In the current study, the similarity in performance accuracy following physical and imagery practices could be due to 
the dominant role of sensory motor predictions made by the forward model for both overt and covert movements. This suggest that in a task where participants had to accurately reproduce previously encoded simple body configurations, motor learning could result from better practice specification for motor command rather than on-line processes or that motor prediction is a fundamental process for both sensorimotor control and learning. In both cases, proprioceptive information would be used off-line to promote more accurate planning of upcoming positions.

\section{Notes}

1. An ANOVA comparing all groups (VP, P, IV, IP and IVP) in transfer tests (after 15 and 150 trials of practice) was performed. Significant main effects of Group, $F(4,49)=8.81, M S E=$ $1.03, p<.001$, and of Test, $F(1,49)=41.69, M S E=0.39, p<.001$, as well as significant interaction between these two factors, $F(1,49)=14.41, M S E=0.39, p<.001$, were observed. Post hoc comparisons showed that, after 150 trials of practice only, RMSE was higher 1) for the VI group than for the other groups $(p<.001)$ and, 2$)$ for the VP group than for the other groups. No difference between groups was observed after 15 trials of practice $(p>.19)$. Moreover, RMSE increased with practice for the IV group $(p<.025)$, decreased for the P, IP and IVP groups $(p<$ $.01)$, while no change appeared for the VP group $(p=.42)$.

\section{References}

Blandin, Y., Toussaint, L., \& Shea, C.H. (2008). Specificity of practice: interaction between concurrent sensory information and terminal feedback. Journal of Experimental Psychology. Learning, Memory, and Cognition, 34, 994-1000.

Binkofski, F., Amunts, K., Stephan, K.M., Posse, S., Schormann, T., Freund, H.J., et al. (2000). Broca's region subserves imagery of motion: A combined cytoarchitectonic and fMRI study. Human Brain Mapping, 11, 273-285.

Coull, J., Tremblay, L., \& Elliott, D. (2001). Examining the specificity of practice hypothesis: Is learning modality specific? Research Quarterly for Exercise and Sport, 72, 345-354.

Decety, J., \& Michel, F. (1989). Comparative analysis of actual and mental movement times in two graphic tasks. Brain and Cognition, 11, 87-97.

Decety, J., Jeannerod, M., Germain, M., \& Pastene, J. (1991). Vegetative response during imagined movement is proportional to mental effort. Behavioural Brain Research, 42, 1-5.

Decety, J., Perani, D., Jeannerod, M., Bettinardi, V., Tadary, B., Woods, R., et al. (1994). Mapping motor representations with positron emission tomography. Nature, 371, 600-602.

Driskell, J.E., Cooper, C., \& Moran, A. (1994). Does mental practice enhance performance? The Journal of Applied Psychology, 79, 481-492.

Féry, Y.A. (2003). Differentiating visual and kinesthetic imagery in mental practice. Canadian Journal of Experimental Psychology, 57, 1-10.

Flanagan, J.R., Vetter, P., Johansson, R.S., \& Wolpert, D.M. (2003). Prediction precedes control in motor learning. Current Biology, 13, 146-150.

Gentili, R., Papaxanthis, C., \& Pozzo, T. (2006). Improvement and generalization of arm motor performance through motor imagery practice. Neuroscience, 137, 761-772.

Georgopoulos, A.P., \& Massey, J.T. (1987). Cognitive spatial-motor processes. Experimental Brain Research, 65, 361-370.

Guillot, A., \& Collet, C. (2005). Duration of mentally simulated movement: a review. Journal of Motor Behavior, 37, 10-20. 
Guillot, A., \& Collet, C. (2008). Construction of the motor imagery integrative model in sport: A review and theoretical investigation of motor imagery use. International Review of Sport and Exercise Psychology, 1, 31-44.

Guillot, A., Collet, C., Nguyen, V.A., Malouin, F., Richards, C., \& Doyon, J. (in press). Brain activity during visual versus kinesthetic imagery: An fMRI study. Human Brain Mapping.

Hall, C.R., \& Pongrac, J. (1983). Movement Imagery Questionnaire. London, Ontario: University of Western Ontario.

Ivens, C.J., \& Marteniuk, R.G. (1997). Increased sensitivity to changes in visual feedback with practice. Journal of Motor Behavior, 29, 326-338.

Jeannerod, M. (1994). The representing brain: Neural correlates of motor intention and imagery. The Behavioral and Brain Sciences, 17, 187-245.

Jeannerod, M. (2001). Neural simulation of action: a unifying mechanism for motor cognition. NeuroImage, 14, 103-109.

Krigolson, O., Van Gyn, G., Tremblay, L., \& Heath, M. (2006). Is there "feedback" during visual imagery? Evidence from a specificity of practice paradigm. Canadian Journal of Experimental Psychology, 60, 24-32.

Mackrous, I., \& Proteau, L. (2007). Specificity of practice results from differences in movement planning and strategies. Experimental Brain Research, 183, 181-193.

Miyai, I., Tanabe, H.C., Sase, I., Eda, H., Oda, I., Konishi, I., et al. (2001). Cortical mapping of gait in humans: a near-infrared spectroscopic topography study. NeuroImage, 14, 1186-1192.

Noel, R. (1980). The effects of visuo-motor behavioural rehearsal of tennis performance. Journal of Sport Psychology, 2, 221-226.

Papadelis, C., Kourtidou-Papadeli, C., Bamidis, P., \& Albani, M. (2007). Effects of imagery training on cognitive performance and use of physiological measures as an assessment tool of mental effort. Brain and Cognition, 64, 74-85.

Proteau, L. (1995). Sensory integration in the learning of an aiming task. Canadian Journal of Experimental Psychology, 49, 113-120.

Proteau, L., \& Carnahan, H. (2001). What causes specificity of practice in a manual aiming movement: vision dominance or transformation errors? Journal of Motor Behavior, 33, 226-234.

Proteau, L., Marteniuk, R.G., Girouard, Y., \& Dugas, C. (1987). On the type of information used to control and learn an aiming movement after moderate and extensive training. Human Movement Science, 6, 181-199.

Proteau, L., Marteniuk, R.G., \& Lévesque, L. (1992). A sensorimotor basis for motor learning: Evidence indicating specificity of practice. Quarterly Journal of Experimental Psychology, 44, 557-575.

Proteau, L., Tremblay, L., \& DeJaeger, D. (1998). Practice does not diminish the role of visual information in on-line control of a precision walking task: Support for the specificity of practice hypothesis. Journal of Motor Behavior, 30, 143-150.

Robin, C., Toussaint, L., Blandin, Y., \& Proteau, L. (2005). Specificity of learning in a video-aiming task: Modifying the salience of dynamic visual cues. Journal of Motor Behavior, 37, 367-376.

Robin, C., Toussaint, L., Blandin, Y., \& Vinter, A. (2004). Sensory integration in the learning of aiming toward "self-defined" targets. Research Quarterly for Exercise and Sport, 74, 381-387.

Robin, N., Dominique, L., Toussaint, L., Blandin, Y., Guillot, A., \& Le Her, M. (2007). Effects of motor imagery training on service return accuracy in tennis: The role of imagery ability. International Journal of Sport and Exercise Psychology, 2, 177-188.

Robin, N., Toussaint, L., \& Blandin, Y. (2006). Importance des modalités d'imagerie dans l'apprentissage de reproduction de configurations corporelles simples. Science \& Motricité, 57, 67-78. 
Roure, R., Collet, C., Deschaumes-Molinaro, C., Delhomme, G., Dittmar, A., \& VernetMaury, E. (1999). Imagery quality estimated by autonomic response is correlated to sporting performance enhancement. Physiology \& Behavior, 66, 63-72.

Shenton, J.T., Schwoebel, J., \& Coslett, H.B. (2004). Mental motor imagery and the body schema: evidence for proprioceptive dominance. Neuroscience Letters, 370, 19-24.

Solodkin, A., Hlustik, P., Chen, E.E., \& Small, S.L. (2004). Fine modulation in network activation during motor execution and motor imagery. Cerebral Cortex (New York, N.Y.), 14, 1246-1255.

Soucy, M.C., \& Proteau, L. (2001). Development of multiple movement representations with practice: specificity versus flexibility. Journal of Motor Behavior, 33, 243-254.

Stinear, C.M., Byblow, W.D., Steyvers, M., Levin, O., \& Swinnen, S.P. (2006). Kinesthetic, but not visual, motor imagery modulates corticomotor excitability. Experimental Brain Research, 168, 157-164.

Stippich, C., Ochmann, H., \& Sartor, K. (2002). Somatotopic mapping of the human primary sensorimotor cortex during motor imagery and motor execution by functional magnetic resonance imaging. Neuroscience Letters, 331, 50-54.

Tremblay, L., \& Proteau, L. (1998). Specificity of practice: the case of powerlifting. Research Quarterly for Exercise and Sport, 69, 284-289.

Tremblay, L., Welsh, T.N., \& Elliott, D. (2001). Specificity versus variability: Effects of practice conditions on the use of afferent information for manual aiming. Motor Control, 5, 347-360.

Wolpert, D.M., \& Kawato, M. (1998). Multiple paire forward and inverse models for motor control. Neural Networks, 11, 1317-1329.

Yue, G., \& Cole, K.J. (1992). Strength increases from the motor program. Comparison of training with maximal voluntary and imagined muscle contractions. Journal of Neurophysiology, 67, 1114-1123.

\section{Appendix A. Specific Guidelines for Imagery Practice}

\section{Visual Imagery:}

"You will be asked to imagine yourself reaching, with your left leg, the angular positions presented during the familiarization session without any movement. For each position, you will focus only on visual information as if you was watching yourself in a mirror located at your left. Each time, you will first imagine to see yourself stretch out your left leg on the position specified by the experimenter, maintain the position for 2 seconds, and bring back your leg to the resting position. Second, you will imagine to see yourself reproduce as accurately as possible the leg displacement and the angular position previously reached."

\section{Proprioceptive (or Kinesthetic) Imagery:}

“... For each position, you will focus only on the 'feel' of the movement leg, in particular the stretch and muscle contraction as well as joint sensation. Each time, you will first imagine to feel yourself stretch out your left leg on the position specified by the experimenter, maintain the position for 2 seconds, and bring back your leg to the resting position. Second, you will imagine to feel yourself reproduce..." 


\section{Visuo-Proprioceptive Imagery:}

“... For each position, you will focus both on visual information as if you was watching yourself in a mirror located at your left and on the 'feel' of the movement leg, in particular the stretch and muscle contraction as well as joint sensation. Each time, you will first imagine to see and feel yourself stretch out your left leg on the position specified by the experimenter, maintain the position for 2 seconds, and bring back your leg to the resting position. Second, you will imagine to see and feel yourself reproduce..." 
Copyright of Motor Control is the property of Human Kinetics Publishers, Inc. and its content may not be copied or emailed to multiple sites or posted to a listserv without the copyright holder's express written permission. However, users may print, download, or email articles for individual use. 